\title{
Surgery for aortic stenosis in severely symptomatic patients older than 80 years: experience in a single UK centre
}

\author{
T Gilbert, W Orr, A P Banning
}

\begin{abstract}
Objective-To ascertain the surgical risk and long term outcome of patients over 80 years old undergoing aortic valve replacement (AVR).

Design-Consecutive cases with respective case note audit and a telephone questionnaire.

Setting-Single UK cardiothoracic surgical centre.

Patients-103 (48 male) patients over 80 years old undergoing AVR. The median age was 82 years (80-95 years) and 95 of 103 patients were in New York Heart Association (NYHA) class III or IV.

Method and results-Preoperative characteristics, operative course, cost, and outcome measures were ascertained. Mean bypass time was 56 minutes and 25 patients had simultaneous coronary artery bypass grafting. Overall mortality was 19 of 103 . Univariate analysis of pertinent variables found that impaired renal function and peripheral vascular disease were significantly associated with early postoperative death. 10 of 12 patients requiring ventilation for more than 24 hours died. The $50 \%$ actuarial survival was 62 months. Late complications were uncommon with $92 \%$ of patients in NYHA class I or II at follow up.

Conclusions-AVR in patients over 80 years old has a significant risk. However, those patients who survive experience significant benefit with good long term prospects for general health and social independence.

(Heart 1999;82:138-142)
\end{abstract}

Keywords: aortic valve replacement; surgical mortality; survival; age

The proportion of very elderly people in the population continues to rise. ${ }^{12}$ Managing these older patients with any medical problem is complex as most prognostic data are based on studies of younger populations and the findings from these studies are not necessarily relevant in the presence of other medical problems. Despite this, aortic valve replacement (AVR) is established as standard management for patients with symptomatic aortic stenosis independent of age. ${ }^{1}$ Selected surgical series suggest that for survivors, successful valve replacement abolishes symptoms and that postoperatively patients have a constant yearly hazard rate that is similar to age and sex matched healthy controls in the general population. $^{3-6}$

Operating on octogenarians has become increasingly commonplace, and for patients with no other concomitant medical problems the decision to offer a patient surgery is usually straightforward. ${ }^{7}$ Combining the published series data suggests a surgical mortality of approximately $10 \%$ for isolated AVR and 20\% for AVR with coronary artery bypass grafting (CABG). However, it is inevitable that within these figures there is both publication and some selection bias towards more healthy patients. This makes interpreting and applying these data for more frail patients with concomitant medical problems complex. It also raises issues of health care provision as elderly patients appear more likely to require prolonged and more intensive use of resources than the younger patients; when funding is limited, issues of resource allocation may arise if the number of patients requiring surgery increases.

We have been aware of increasing numbers of very elderly patients with aortic stenosis presenting to our centre with severe symptoms. Our centre has no predetermined surgical criteria for patient selection and each case is managed on an individual basis. We have retrospectively reviewed our 10 year consecutive experience of aortic valve surgery in patients over 80 years old. Review of case notes was followed by a telephone questionnaire of the patients' general practitioners. Our objectives were to obtain a local estimate of surgical risk for patients $>80$ years old requiring AVR, to determine which risk factors were pertinent in our practice in predicting a poor outcome and to assess whether the increasing numbers of patients likely to be considered for such surgery in the future were likely to have significant financial and logistic implications.

Methods

Between January 1987 and December 1996, 103 AVRs were performed on patients of at least 80 years of age in a single large UK cardiothoracic centre. Cases were consecutive and all hospital records were reviewed to obtain information about preoperative characteristics and operative and postoperative details. One hundred and two of 103 case notes were available for review. Follow up data were obtained from clinic letters and by telephone conversation with the patients' general practitioners. The patients' general health, functional status (New York Heart Association (NYHA) class), social 
circumstances, number of admissions to hospital since their operations, and medication were ascertained. Dates and causes of death were established using hospital records and data obtained from the Office for National Statistics.

PREOPERATIVE INVESTIGATION

Transthoracic echocardiography including Doppler was performed using standard techniques. Systolic function was estimated visually by experienced observers and graded as normal, mildly/moderately impaired, or severely impaired. Peak instantaneous valvar gradients were obtained using continuous wave Doppler and valve area was estimated using the continuity equation. The degree of aortic reflux was assessed using colour Doppler and standard echocardiographic criteria.

Left heart catheterisation was performed from the right femoral artery according to standard clinical protocols, with single plane coronary angiography and aortic root angiography. Left ventricular angiography and measurement of peak instantaneous gradient was only made in cases where there was uncertainty about the severity of the valvar stenosis. Coronary artery disease was defined as any lesion in a coronary artery $>50 \%$ of the luminal diameter in one view. Patients considered too unwell to be considered for coronary angiography were referred directly for an AVR.

Renal impairment was classified by a serum creatinine of $\geqslant 140 \mathrm{mmol} / \mathrm{l}$. Heart failure was determined by evidence of pulmonary congestion on the chest radiograph.

\section{SURGICAL PROCEDURE}

Aortic valve surgery was conducted through a midline sternotomy using standard techniques. In general, surgical policy was to minimise aortic cross clamp time and the duration of cardiopulmonary bypass, particularly in very sick patients. Crystalloid anterograde cardioplegia was used but the patients were not systemically cooled. CABG was performed when indicated during the same procedure based on a decision made by the surgeon in liaison with the cardiologist. Patients considered to be too unwell for cardiac catheterisation underwent valve replacement alone. Postoperative care was initially conducted on the cardiac recovery unit, but if patients required prolonged ventilation or renal support they were transferred to intensive care unit.

\section{COSTING}

The contracted price to purchasers for AVR in our trust is calculated from an expected period of 12 hours in the cardiac recovery and five days postoperative ward care. The contract prices for our trust for 1997-98 were used for the calculations in this paper and are available from the authors. Urgent surgery was categorised as surgery performed for immediately life threatening symptoms or for patients too unwell to be discharged from hospital before operation. Early mortality was defined as death within 30 days of surgery.

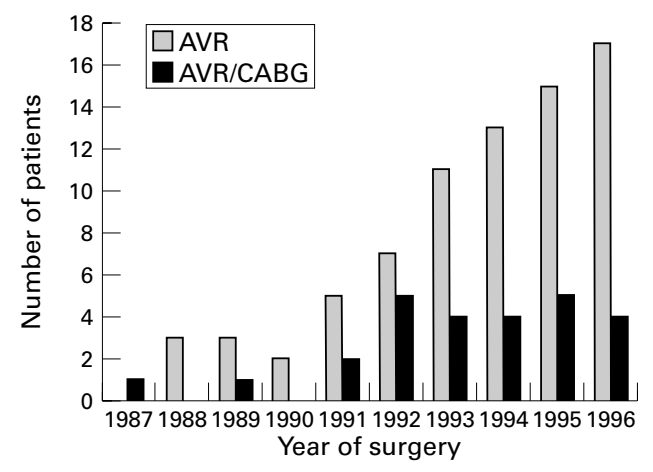

Figure 1 Temporal trend of patients more than 80 years old undergoing $A V R$ and concomitant $C A B G$ between 1987 and 1996.

\section{STATISTICS}

Statistical analysis was performed using Statview 4.5 (Abacus Concepts). Risk factor analysis was performed in univariate fashion and by means of contingency tables and $\chi^{2}$ methods. Actuarial survival curves were calculated, as were proportional hazard models.

\section{Results}

PATIENT CHARACTERISTICS

Over the study period there was an annual increase in the number of patients aged 80 or over having surgery for aortic stenosis (fig 1). There were 55 women and 48 men with a mean (SD) age of 83 (2.6) years (range 80-95 years). The most common principal symptom was dyspnoea (95 of 103 patients) and $92 \%$ of patients were NYHA class III or IV. Twenty one patients had exertional syncope and 19 had exertional chest pain. Symptoms had been present in 47 patients for $>12$ months, in 31 patients for 3-12 months, and in 23 patients for $<3$ months.

Comorbid conditions included hypertension ( $n=19$ ), previous neurovascular disease $(n=9)$, peripheral vascular disease $(n=6)$, and preoperative atrial fibrillation $(n=6)$. Twenty three patients had impaired renal function. There was one diabetic and one patient had a previously documented myocardial infarction. Three patients had undergone previous cardiac surgery and one patient had previously undergone aortic balloon valvuloplasty. The mean calculated Parsonnet score for our patient group was 30 (range 25-47).

Results of preoperative echocardiography were available in 99 patients. Left ventricular function was subjectively impaired in $52 \%$. The mean (SD) peak instantaneous aortic valve gradient was 86 (27) $\mathrm{mm} \mathrm{Hg}$. Twenty nine $(28 \%)$ patients had mild to moderate or greater aortic reflux. No patients had significant mitral valve disease.

Cardiac catheterisation was carried out in 86 (84\%) patients. Coronary disease was present in 35 of these patients: 13 patients had one vessel disease, 10 had two vessel disease, and 12 had three vessel disease. Patients with coronary disease primarily complained of syncope $(n=5)$, shortness of breath $(n=20)$, or chest pain $(n=10)$. Patients with no significant coronary disease primarily complained of syncope 
Table 1 Preoperative characteristics and relation to operative mortality

\begin{tabular}{lll}
\hline Characteristic & Number $(n=103)$ & $\begin{array}{l}\text { Significance level of } \\
\text { predictor ( } p \text { value) }\end{array}$ \\
\hline Mean age (range 80-95) (years) & 83 & 0.01 \\
Female:male & $55: 48$ & $\mathrm{NS}$ \\
NYHA class III-IV & 95 & $\mathrm{NS}$ \\
Diabetes & 1 & $\mathrm{NS}$ \\
Chronic airways disease & 7 & $\mathrm{NS}$ \\
Previous stroke/transient ischaemic attack & 9 & $\mathrm{NS}$ \\
Preoperative atrial fibrillation & 6 & $\mathrm{NS}$ \\
Preoperative permanent pacemaker & 3 & $\mathrm{NS}$ \\
Peripheral vascular disease & 6 & 0.002 \\
Renal impairment & 23 & 0.001 \\
Impaired left ventricular function & 51 & $\mathrm{NS}$ \\
Coronary disease & 35 & $\mathrm{NS}$ \\
Simultaneous CABG & 25 & $\mathrm{NS}$ \\
Previous cardiac operation & 3 & $\mathrm{NS}$ \\
\hline
\end{tabular}

NS, not significant.

( $n=11)$, shortness of breath $(n=30)$, or chest pain $(\mathrm{n}=10)$.

MORTALITY AND MORBIDITY

One hundred and three patients underwent surgery which was successfully completed in all patients without any major intraoperative difficulties. In one patient an intra-aortic balloon pump was necessary to wean the patient from cardiopulmonary bypass; this patient subsequently died of multiple organ failure.

Twenty five patients had simultaneous CABG and AVR; 12 patients had a single graft, while 13 had two or more grafts. Early mortality was $20 \%$ (5 of 25). There was no significant difference in mortality following concomitant CABG and AVR compared with valve surgery alone ( $p>0.8)$. Mean cardiopulmonary bypass time was 56 minutes and the mean aortic cross clamp time was 45 minutes. The mean bypass time in patients not receiving coronary grafts was 50 minutes $v 74$ minutes in those patients who underwent grafting $(\mathrm{p}<0.0001)$. There was no significant difference in cross clamp or bypass times for survivors compared with early postoperative death. Neither CABG nor the need for emergency surgery were predictors of an adverse outcome.

Ten patients with coronary disease did not undergo CABG. This decision was made to reduce total bypass time in critically unwell patients except for one patient who had undergone previous $\mathrm{CABG}$ and grafts were patent. Two of three patients with left anterior descending artery disease who did not have coronary grafts subsequently died. The other six patients with coronary disease who did not undergo concomitant surgery survived; one patient had two vessel disease and the others had single vessel coronary disease. Overall the early mortality of patients with identified coronary disease undergoing AVR was 23\% (8 of 35).

Carpentier Edwards porcine bioprostheses $(n=61)$ were used for the majority of patients, but towards the end of the study period increasing numbers of patients received either a Medtronic $(\mathrm{n}=21)$ or a Baxter $(\mathrm{n}=4)$ stentless valve. Seven patients had a pericarbon prosthetic valve.

Seventeen patients underwent valve replacement without preoperative cardiac catheterisa-
Table 2 Postoperative complications

\begin{tabular}{ll}
\hline Complication & $n(\%)$ \\
\hline Atrial fibrillation & $26(25)$ \\
Stroke & $17(17)$ \\
Reopening for bleeding & $4(4)$ \\
Renal support & $13(12)$ \\
Left ventricular failure & $6(6)$ \\
Permanent pacemaker & $11(11)$ \\
\hline
\end{tabular}

tion; five patients complained of syncope, 10 of shortness of breath, and two of chest pain. Early mortality following surgery in this group was $12 \%$ (2 of 17) and was similar to the group as a whole.

Postoperatively, all patients returned to the cardiac recovery unit. Five patients required ventilation for more than 48 hours and they were transferred to the general intensive care unit. Only two of the 12 patients requiring ventilation for more than 24 hours survived $>30$ days postoperatively. The duration of postoperative ventilation and time spent on intensive care were therefore inevitably greater in those who died in the early postoperative period compared with survivors. No pre- or perioperative predictors for prolonged ventilation or intensive treatment were identified except the use of renal support - that is, dopamine/ haemofiltration or dialysis in those patients who died early. Eight of 13 patients requiring renal support died early while only 11 of 89 not requiring renal support died.

Thirty four patients had urgent surgery. For the group as a whole, early mortality was $19 \%$ (19 of 101). Factors thought to contribute to death were intractable left ventricular failure $(n=5)$, cerebral event $(n=6)$, myocardial infarction $(n=3)$, renal failure $(n=2)$, aortic dissection $(\mathrm{n}=1)$, small bowel infarction $(n=1)$, multiple organ failure $(n=2)$, and fatal cardiac arrhythmia $(n=1)$.

The only significant determinants of early postoperative mortality (table 1 ) were increasing age $(p=0.0121)$, renal impairment $(p=0.0014)$, and peripheral vascular disease $(\mathrm{p}=0.0017)$. Impaired left ventricular function, presence of coronary disease, coronary grafting, and repeat surgery did not appear to influence outcome.

Sixty patients suffered at least one postoperative complication (table 2). Seventeen had cerebrovascular events of which six contributed to early mortality $(p=0.05)$ and a further two patients died within six months. Five of the patients with stroke made full recoveries. Although numbers were small a history of claudication $(n=6)(p<0.03)$ and increasing age $(p=0.05)$ correlated with postoperative cerebral event.

LONG TERM FOLLOW UP

Information on long term follow up was obtained from the general practitioners who had all seen the patients $(n=64)$ at least once within the preceding three months. Mean survival after surgery was 62 months with a $50 \%$ actuarial survival of 67 months (fig 2). This compares favourably with unselected 80 year old people and it is considerably better than for patients with severe aortic stenosis who have 


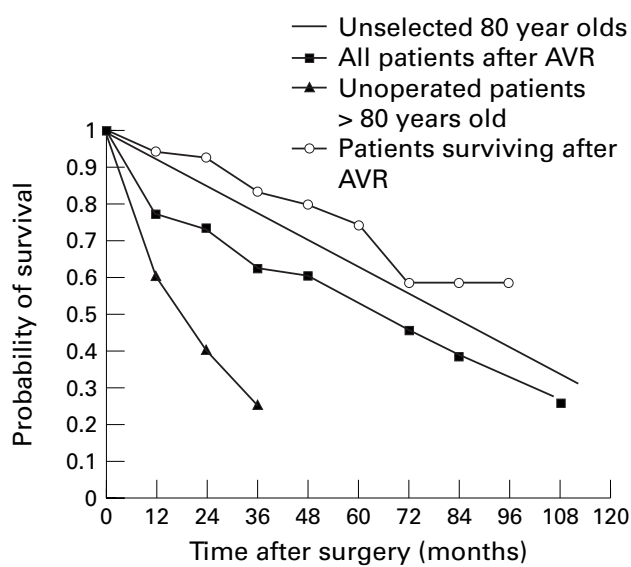

Figure 2 Comparison of outcome between our patients and historical controls. Data are shown for an unselected population of 80 year olds in the US ${ }^{3}$ and patients over 80 years old with symptomatic aortic stenosis ${ }^{8}$ (solid triangles). Data from our study has been superimposed to show both our total population (solid boxes) and the long term survival for patients surviving $>30$ days postoperatively (open circles).

not had surgery. Ninety two per cent of long term survivors were NYHA class I or II, compared with $92 \%$ who were NYHA class III or IV preoperatively.

Preoperatively 53 subjects were living at home with their partners, 23 alone and self caring, 22 lived alone with social support (either family, local government or both), and three were in nursing homes. Of the 86 patients who left hospital following their surgery, 36 were discharged home, 25 transferred to district general hospital, and 25 to community hospitals or nursing homes. Fifty patients were transferred to other hospitals or nursing homes before being discharged home. The median stay in these institutions was 12 days (range 1-255 days). At follow up 56 of 64 patients were still living at home alone, alone with social support, or with partners.

Late complications following surgery were uncommon. Nineteen of the 84 survivors required admission to hospital but in only nine patients was this related to the cardiovascular system (six congestive cardiac failure, two complete heart block, and one endocarditis). Of the 63 patients still alive at follow up 52 were taking one or more different forms of medication. The mean number of drugs per live patient was 2.13 . The most commonly prescribed drugs among the 63 live patients were diuretics (46), warfarin (19), aspirin (18), digoxin (18), and angiotensin converting enzyme inhibitors (10).

\section{COSTINGS}

The median stay on the cardiac recovery ward was 20 hours compared with an expected stay of 12 hours. Intensive care was not budgeted for in the contracted price but the observed median time spent was 14.5 hours. The expected mean figure of five days postoperative ward care was exceeded by three days. This observed pattern of care resulted in an increased mean cost per patient of $50 \%$ above the contracted price.

\section{Discussion}

This study documents the results of AVR in patients $>80$ years old in a large single surgical centre. The overall operative risk was $18 \%$ compared to an expected risk calculated from the Parsonnet score of $30 \%$. Impaired renal function and particularly peripheral vascular disease were identified as predictive factors suggesting a poor outcome and a high perioperative risk. Our patients were high risk candidates, being severely symptomatic with a high incidence of coronary disease. Despite their parlous preoperative state, survivors of surgery experienced dramatic improvements in symptoms and few complications or illnesses following discharge, and many were able to resume independent lives in the community.

The overall operative risk in this series was $18 \%$, which is slightly higher than the operative risk derived from the literature of $14 \%$ and significantly higher than the results of the recently published UK heart valve registry $(7 \%),{ }^{9}$ in which our centre participates. We speculate that the reasons for this difference could include the severe symptomatic level of our patients preoperatively (95 of 105 in NYHA class III or IV), the high level of renal and coronary disease, and perhaps most importantly the lowering of our threshold for considering surgery in these very elderly patients, particularly those with concomitant medical problems. Overall we feel that these operative risks are perhaps more realistic for the general population than some of the previously published series.

This study illustrates the complexity of anginal pain in patients with aortic stenosis. Of the 86 patients who underwent coronary angiography, 17 had anginal type chest pain as their principal symptom. Eight patients had significant coronary disease in one or more vessels whereas nine had no significant coronary disease. The mechanisms of anginal pain in this group of patients with normal coronary arteries are complex but they include left ventricular hypertrophy and impaired diastolic function in association with a shortened diastolic perfusion time. ${ }^{10}$

A subgroup of patients in this study $(n=17)$ underwent valve replacement without preoperative cardiac catheterisation. These patients were all in congestive cardiac failure at the time of surgery despite medical treatment, although five patients also complained of syncope and two patients had experienced chest pain. Early mortality following surgery in this group was similar to the group as a whole despite their limited preoperative assessment. Despite this we continue to advocate coronary angiography for all of our patients before valve surgery if the procedure can be performed with an acceptable risk, and increasingly we perform coronary angiography on the way to theatre occasionally with intra-aortic balloon pump support.

Impaired left ventricular systolic function was not identified as an adverse prognostic factor in this series. Conventionally in the absence of coronary disease, left ventricular systolic dysfunction is not considered a contraindication to surgery as it is likely that ventricular 
function will improve following relief of the valvar obstruction. In our unit, these patients are all referred for surgery unless there are mitigating factors. Judging the relative contributions of valvar disease and ischaemic heart disease to left ventricular systolic dysfunction in a patient with coronary disease is extremely complex. Previously the tendency has been to recommend surgery and revascularisation but increasingly we are utilising dobutamine stress echocardiography to augment our assessment.

Symptomatic peripheral vascular disease and increasing age both predicted stroke. This affected $17 \%$ of patients which is a higher rate than most other published series. In general, our surgical policy has been to minimise the duration of surgery and the bypass time. Culliford et al commented on their low incidence of stroke $(1.4 \%)$ and cited the fact that in patients with heavily calcified valves a long arch arterial return cannula was used and that meticulous attention was given to the removal of calcific valvar debris and air. ${ }^{5}$ Other series have suggested that aortic calcification, older age, perioperative hypotension, and prolonged bypass time ${ }^{11}$ are important determinants of stroke, and that systemic hypothermia may be more likely to be more protective for the brain than normothermia. ${ }^{12}$

Based on the results of this study we are currently considering whether we should adjust our practice in this elderly age group, including use of transoesophageal echocardiography to assess aortic atheroma.

Patients requiring prolonged ventilation and intensive treatment in this series were unlikely to recover and no patient ventilated for more than 36 hours survived. This was not predictable from preoperative factors, and patients with chronic pulmonary disease were no more likely to require prolonged ventilation. Haemodynamic instability, renal insufficiency, and persistent pulmonary oedema were reasons for prolonged ventilation. Although these data could be valuable in the future when considering whether to continue prolonged supportive treatment in an individual, the failure of preoperative factors to identify which patients are likely to fall into this category limits its applicability.

In common with a number of previous authors we have shown excellent long term outcome for the survivors of surgery. ${ }^{6}{ }^{13}$ Most survivors were able to return to similar social circumstances to their preoperative arrangements although many needed prolonged rehabilitation. The low rates of hospital admission following surgery are particularly gratifying as without surgery many of these patients would have required continuing intensive nursing support, and they would never have been able a return to a self caring environment.

We have shown that these very elderly patients do use more resources than are currently budgeted for. Other comparative studies with younger patients support this conclusion..$^{74}$ This excess cost is in part a reflection of the expense incurred by patients admitted to intensive care, but there is not surprisingly an increased inpatient period for all of these more elderly patients, even when surgery is uncomplicated. In the context of the increasing proportion of older patients in the community, these factors must be recognised when planning health care strategies and preparing budget provision.

CONCLUSIONS

This study demonstrates that AVR in patients $>80$ years old is associated with a significant operative risk, particularly in the presence of peripheral vascular disease or impaired renal function. However, successful surgery offers survivors good long term prospects for general health and social independence, and despite the cost of surgery this beneficial outcome could result in long term health care savings. Continuing evolution of surgical and anaesthetic techniques will reduce operative risks and in elderly patients this may result in more patients being eligible for surgery. Selecting which patients are likely to benefit remains a challenge for both the cardiologist and the cardiothoracic surgeon.

The authors are grateful to Dr Brian Gribbin, the other cardiologists, Mr Steve Westaby, and Mr Ravi Pillai for their permission to report these data. We also acknowledge the contribution of nursing and technical staff to patient care.

1 Sprigings DC, Forfar JC. How should we manage symptomatic aortic stenosis in the patient who is 80 years or older? Br Heart $\mathcal{F}$ 1995;74:481-4.

2 Lindroos M, Kupari M, Heikkila J, et al. Prevalence of aortic valve abnormalities in the elderly: an echocardiographic tic valve abnormalities in the elderly: an echocardiographic study of a random
$1993 ; 21: 1220-5$.

3 Levinson JR, Akins CW, Buckley MJ, et al. Octagenarians with aortic stenosis: outcome after aortic valve replacement. Circulation 1989;80(suppl I):49-56.

4 Fiore AC, Naunheim KS, Barner HB, et al. Valve replacement in the octagenarian. Ann Thorac Surg 1989;48: 104-8.

5 Culliford AT, Galloway AC, Colvin SB, et al. Aortic valve replacement for aortic stenosis in persons aged 80 years and over. Am f Cardiol 1991;67:1256-60.

6 Gehlot A, Mullany CJ, Ilstrup D, et al. Aortic valve replacement in patients aged eighty years and older: early and long-term results. F Thorac Cardiovasc Surg 1996;111: 1026-36.

7 Edmunds LH Jr, Stephenson LW, Edie RN, et al. Open-heart surgery in octagenarians. $N$ Engl $f \mathrm{Med}$ Open-heart surger

8 O'Keefe JH, Vliestra RE, Bailey KR, et al. Natural history of candidates for balloon aortic valvuloplasty. Mayo Clin Proc 1987;62:986-91.

9 Asimakopoulous G, Edwards MB, Taylor KM. Aortic valve replacement in patients 80 years of age and old. Survival and cause of death based on 1100 cases: collective results from the UK heart valve registry. Circulation 1997;96: 3403-8.

10 Gould KL. Why angina pectoris in aortic stenosis. Circulation 1997;95:790-2.

11 Garner TJ, Horneffer PJ, Manolio TA, et al. Stroke following coronary bypass grafting: a 10 year study. Ann Thorac Surg 1985;97:574-81.

12 Martin TD, Craver JM, Gott JP, et al. Prospective randomized trial of retrograde warm blood cardioplegia: myocardial benefit and neurologic threat. Ann Thorac Surg 1994;57:298-304

13 Olsson $M$, Janfjall $\mathrm{H}$, Orth-Gomer $\mathrm{K}$, et al. Quality of life after valve replacement due to aortic stenosis. Eur Heart 7 1996;17:583-9.

14 Olsson M, Granstrom L, Lindblom D, et al. Aortic valve replacement in octagenarians with aortic stenosis: a case control study. $\mathcal{F}$ Am Coll Cardiol 1992;20:1512-16. 